\title{
Epilepsy in Primary Cerebral Tumors: Long-Term Follow- Up of Seizures in the PERNO Study (Project of Emilia Romagna Region on Neuro-Oncology)
}

\author{
Pasini $E^{1 *}$, Fallica $E^{2}$, Monetti $C^{2}$, Meletti $S^{3}$, Monti $G^{3}$, Florindo $I^{4}$, Rizzi $R^{5}$, Michelucci $R^{6}$ and \\ The PERNO Study Group
}

${ }^{1}$ Department of Biological and Neuromotor Sciences, University of Bologna, Italy

${ }^{2}$ Unit of Neurology, University Hospital, Ferrara, Italy

${ }^{3}$ Department of Biomedical Sciences, Metabolism and Neuroscience, University of Modena and Reggio Emilia, Modena, Italy

${ }^{4}$ Unit of Neurology, Department of Neurosciences, University of Parma, Parma, Italy

${ }^{5}$ Unit of Neurology, Department of Neuromotor Physiology, IRCCS - Santa Maria Nuova Hospital, Reggio Emilia, Italy

${ }^{6}$ Unit of Neurology, IRCCS- Institute of Neurological Sciences of Bologna, Bellaria Hospital, Bologna, Italy

\begin{abstract}
Objective: Despite the high prevalence of epilepsy in brain tumors, long-term follow-up studies on tumoral epilepsy are lacking. Here we report the long-term follow-up results of the PERNO study (Project of Emilia-Romagna Region on NeuroOncology).

Methods: PERNO study enrolled for a three-year period every person living in Emilia-Romagna Region with a new diagnosis of primary brain tumor (PBT). We previously described the short-term results of epilepsy evolution after the first surgical procedure in 100 patients; for 52 patients of this group, the long-term results of seizure outcome after a mean follow-up of 24 months are now available.

Results: Out of these 52 patients, 41 presented with a high-grade glioma (HGG), whereas 11 had a low-grade glioma $(L G G)$ at the onset of the disease (HGG/LGG ratio $=3.7$ ) and were followed-up for a median period of 548 and 1032 days, respectively. The HGG group showed a seizure-freedom rate of more than $60 \%$, with a better seizure control (up to $87 \%$ ) being achieved in patients without evidence of tumor progression. Conversely, in the LGG group complete seizure control occurred only in one case (less than $10 \%$ of patients), with most patients requiring surgical revision due to the extension of the tumor.

Conclusions: Our data show that epilepsy associated with HGG seems to have a relatively good outcome, especially if the tumor is stable. In contrast, seizure control in LGG was more difficult to be achieved, suggesting the need of detailed epileptological study (including long-term video-EEG monitoring) before surgery to improve seizure control.
\end{abstract}

Keywords

Epilepsy, Cerebral tumors, High grade gliomas, Low grade glioma, Long-term follow-up

\section{Introduction}

Epilepsy is a common symptom of primary brain tumors (PBT), its prevalence ranging between 30 and $100 \%$ as a result of tumor histology and location [1]. While low-grade gliomas (LGG) are highly epileptogenic and are associated with a relatively long survival time, high-grade gliomas (HGG) are characterized by lower incidence of seizures and a short survival time [1].

Therefore, most studies addressing long-term follow-up of tumoral epilepsy focused on LGG. We previously published
*Corresponding author: Elena Pasini, Department of Biological and Neuromotor Sciences, University of Bologna, Via Altura, 3 Bologna 40139, Italy, Tel: 0039/3387014122

Accepted: June 12, 2021

Published online: June 14, 2021

Citation: Pasini E, Fallica E, Monetti C, et al. (2021) Epilepsy in Primary Cerebral Tumors: Long-Term Follow-Up of Seizures in the PERNO Study (Project of Emilia Romagna Region on NeuroOncology). J Brain Tumor 1(1):1-5 
Citation: Pasini E, Fallica E, Monetti C, et al. (2021) Epilepsy in Primary Cerebral Tumors: Long-Term Follow-Up of Seizures in the PERNO Study (Project of Emilia Romagna Region on Neuro-Oncology). J Brain Tumor 1(1):1-5

the short-term follow-up of seizures in a group of patients who were enrolled in the PERNO study (Project of Emilia Romagna Region on Neuro-Oncology), a prospective registry of PBTs in Emilia Romagna Region (Italy), showing that seizures were responsive to surgical treatment in $60 \%$ of patients, who became seizure free after surgical procedure, irrespective of tumor type and presurgical seizure frequency [2].

Now we present the long-term follow-up data of this group of patients, with special emphasis on seizure evolution.

\section{Materials and Methods}

This study is the long-term follow-up of the epilepsy section of the PERNO study [2]. In particular, in the epilepsy section of PERNO study we enrolled all new adult cases living in the Emilia Romagna Region with suspected PBT observed from January 2009 until December 2011 who experienced seizures, either as first symptom of the tumor or appearing during the course of the disease. Each patient was interviewed by the referring neurologist with a specific interest in epilepsy who used an ad hoc questionnaire to collect information about seizure semiology, neurologic status, tumor location and histology, and treatments (including antiepileptic regimen, chemotherapy, radiotherapy, and surgery).

After the first visit, the patients who entered the longterm study were followed up with repeat visits on a quarterly basis, until February 2014. At each evaluation, the clinical data and seizure characteristics were recorded, along with any change in therapy (antiepileptic and antineoplastic) and new neuro-imaging findings. When the patients could not come to visits, clinical information was collected by phone interview with the referring neurologist. A consent form was obtained by each patient or responsible guardian.

Statistical analysis was performed by Chi-square test and Fisher's exact test. Seizure outcome was assessed using categories of change during follow-up (seizure freedom; $>75 \%$ reduction; no difference; increase). The cumulative probability to remain seizure-free was calculated using Kaplan-Meier survival curves. Significance was set at the $5 \%$ level $(p=0.05)$.

\section{Results}

Out of the 100 patients with PBT associated with epilepsy (62 male and 38 female, mean age 47 years) participating in the previous short-term study, 48 were lost at follow-up, due to a number of reasons, including lack of multidisciplinary management, hospitalization in other institutions, and rapid neurological deterioration. The remaining 52 patients were followed up until December 312012 and their characteristics are described below.

\section{Tumor type and location}

The patients were followed for 104.9 person-years HGG accounted for the vast majority of patients, with a $3.7: 1$ ratio. 41 patients (mean age at the onset 53 years) had HGG (glioblastoma in 23 cases), whereas 11 patients had low-grade lesions including astrocytomas, oligodendrogliomas and oligoastrocytoma (mean age at the onset 33 years) (Table 1 ). Median follow-up in HGG and LGG was 548 (IQR 329-847) and 1032 (IQR 692-1394) days, respectively. The most common tumor location was frontal $(69.2 \%$ overall, $90.9 \%$ in LGG, $63.4 \%$ in $\mathrm{HGG}$ ), temporal (36.5\% overall, $54.5 \%$ in LGG, $31.7 \%$ in $\mathrm{HGG}$ ) and parietal ( $25 \%$ overall, $27.3 \%$ in LGG and $24.4 \%$ in HGG). Multiple lobes were involved in $48.1 \%$ of cases $(54.5 \%$ in LGG and $46.3 \%$ in HGG).

\section{Tumor prognosis}

Within the HGG group only 8 patients (19.5\%) had stable MRI findings and neurological status. Of the remaining 33 patients $(80.5 \%) 22$ died and 11 showed tumor progression at cerebral MRI during the follow-up.

In the LGG group, 10 of 11 patients underwent surgery: 5 patients were stable on repeat cerebral MRI, 5 needed further surgical revision and 1 deceased. Of the 5 patients who underwent surgical revision, 2 underwent revision three times and 3 patients one time, while histology showed a progression into HGG in 2 cases.

\section{Seizures}

Seizure semiology did not substantially change during disease evolution as compared to the early phase of the disease (Table 2). As previously reported, the majority of seizures were tonic clonic (38.5\%) (without clear initial focal signs in more than one half of the patients), focal motor in $28.8 \%$ of patients and focal somato-sensitive in $15.4 \%$ of cases.

Seizure frequency in relation to tumor grade and progression is reported in (Figure 1a) (HGG) and (Figure 1b) (LGG). The cumulative probability of remaining seizure-free in patients with HGG was $100 \%$ at 12 months and $100 \%$ at 24 months (Figure 2). The corresponding values for patients with LGG were $87 \%$ (95\% Cl 71-94\%) and 60\% (95\% Cl 41-75\%). In the HGG group, $63 \%$ of patients were seizure free on longterm follow-up (median time: 547 days, IQR 400-778), a rate comparable with that observed after initial surgical removal. Status epilepticus did not substantially change during the follow-up period, with a frequency of $9.8 \%$ (4 patients, all during tumor progression). In the group of patients without tumor progression, seizure freedom was reached in 7 of 8 cases, whereas in the two groups with tumor worsening (as demonstrated either by imaging or evolution to death) seizure freedom was achieved in 7 of 22 and 6 of 11, respectively.

Table 1: Histologic type and grade of tumors.

\begin{tabular}{|l|l|l|}
\hline Tumor grade & Tumor type & $\mathbf{N}^{\circ}$ \\
\hline II & Astrocytoma & 3 \\
\hline III & Anaplastic astrocytoma & 8 \\
\hline IV & Glioblastoma & 23 \\
\hline II & Oligodendroglioma & 6 \\
\hline III & Anaplastic oligodendroglioma & 5 \\
\hline II & Mixed oligoastrocytoma & 1 \\
\hline III & Anaplastic oligoastrocytoma & 2 \\
\hline No surgery & & 4 \\
\hline Total & & 52 \\
\hline
\end{tabular}


Citation: Pasini E, Fallica E, Monetti C, et al. (2021) Epilepsy in Primary Cerebral Tumors: Long-Term Follow-Up of Seizures in the PERNO Study (Project of Emilia Romagna Region on Neuro-Oncology). J Brain Tumor 1(1):1-5
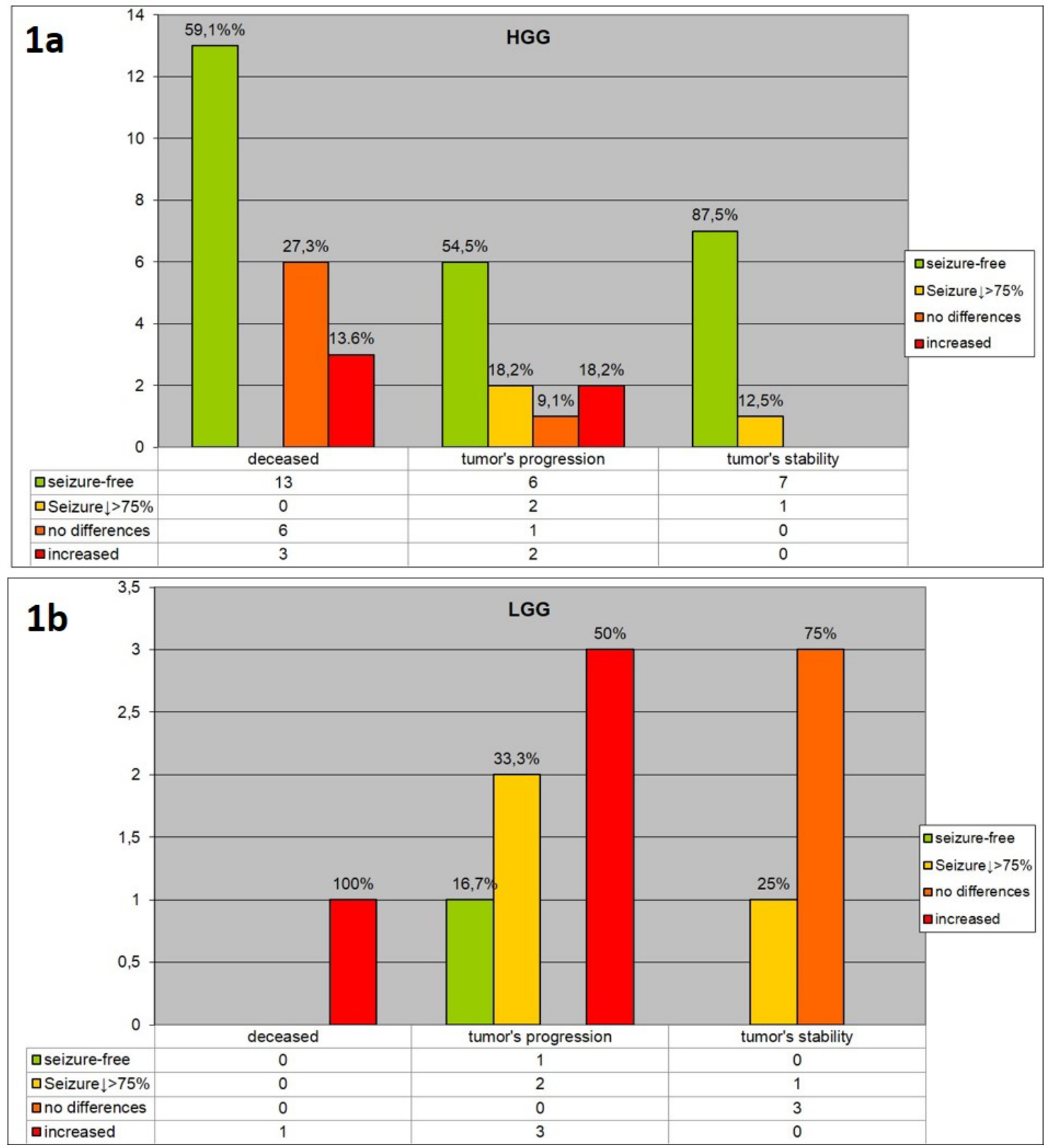

Figure 1: Seizure outcome in relation to tumor progression in HGG (1a) and LGG groups (1b).

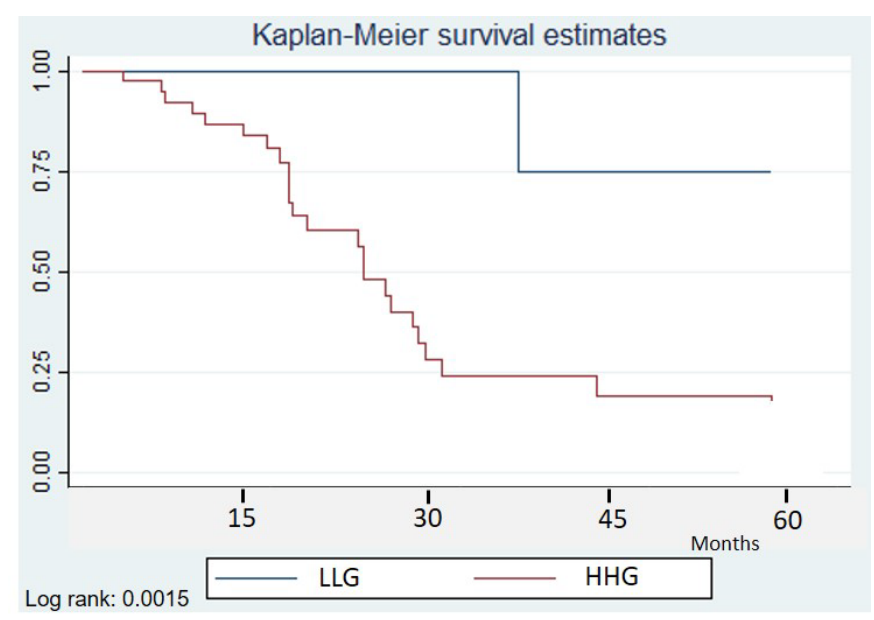

Figure 2: Cumulative time-dependent probability of remaining seizure-free in HGG and LGG groups. 
Citation: Pasini E, Fallica E, Monetti C, et al. (2021) Epilepsy in Primary Cerebral Tumors: Long-Term Follow-Up of Seizures in the PERNO Study (Project of Emilia Romagna Region on Neuro-Oncology). J Brain Tumor 1(1):1-5

Table 2: Comparison of baseline features between study population and patients lost at follow-up.

\begin{tabular}{|c|c|c|}
\hline & $\begin{array}{l}\text { Long-term follow-up } \\
\text { study } 52 \text { patients (\%) }\end{array}$ & $\begin{array}{l}\text { Patients lost at long- } \\
\text { term follow-up } 48 \\
\text { patients (\%) }\end{array}$ \\
\hline Age & $\begin{array}{l}49 \text { years ( } \max 76, \\
\min 19)\end{array}$ & $\begin{array}{l}57 \text { years (max } 76, \\
\min 19)\end{array}$ \\
\hline Sex (M:F) & $2.1(35 / 17)$ & $1.3(27 / 21)$ \\
\hline Histology (HGG/LGG) & $3.7(41 / 11)$ & $3.6(36 / 10)$ \\
\hline \multicolumn{3}{|l|}{ Tumor location } \\
\hline Frontal & $36(69.2 \%)$ & $24(50.0 \%)$ \\
\hline Temporal & 19 (36.5\%) & 18 (38.5\%) \\
\hline Parietal & $13(25.0 \%)$ & $14(29.2 \%)$ \\
\hline Occipital & $5(9.6 \%)$ & $5(10.4 \%)$ \\
\hline Insula & $6(11.5 \%)$ & $2(4.2 \%)$ \\
\hline Other & $6(11.5 \%)$ & $6(12.5 \%)$ \\
\hline Multilobar & 25 (48.1\%) & $12(25.0 \%)$ \\
\hline \multicolumn{3}{|l|}{ Seizure type } \\
\hline Motor & $15(28.8 \%)$ & $16(33.3 \%)$ \\
\hline Somato-sensory & $8(15.4 \%)$ & $3(6.3 \%)$ \\
\hline Loss of consciousness & $10(19.2 \%)$ & $10(20.8 \%)$ \\
\hline Other & $8(15.4 \%)$ & $4(8.3 \%)$ \\
\hline Tonic-Clonic & $20(38.5 \%)$ & 25 (52.1\%) \\
\hline \multicolumn{3}{|l|}{ Frequency } \\
\hline Low (<1/Month) & 31 (59.6\%) & 37 (71.2\%) \\
\hline High (> 1/Month) & $21(40.4 \%)$ & 11 (22.9\%) \\
\hline Weekly & $8(15.4 \%)$ & 0 \\
\hline Daily & $9(17.3 \%)$ & $1(2.0 \%)$ \\
\hline Status epilepticus & $4(7.7 \%)$ & $8(16.7 \%)$ \\
\hline \multicolumn{3}{|l|}{ Surgery } \\
\hline Radical surgery & $13(25.0 \%)$ & 25 (52.1\%) \\
\hline Post-surgery residue & $33(63.5 \%)$ & $20(41.6 \%)$ \\
\hline \multicolumn{3}{|l|}{ General prognosis } \\
\hline Dead & $23(44.2 \%)$ & 21 (43.8\%) \\
\hline
\end{tabular}

In the LGG group only 1 patient (9.1\%) remained seizure free during follow-up (1153 days), despite tumor progression to glioblastoma. Conversely, in this group 1 patient had epilepsy worsening evolving to status epilepticus during tumor relapse and needed surgical revision.

\section{Medical treatment}

All patients were on medical treatment for epilepsy. The most common antiepileptic drug was levetiracetam (LEV) (65\%), that prevailed in the HGG group (73.2\%); in contrast, oxcarbazepine (OXC) was chosen for $81.8 \%$ of patients belonging to the LGG group.

Monotherapy was prescribed in 28 patients (53.8\%), whereas 20 patients (38.5\%) were on two drugs and only 4 (7.7\%) on a three drug regimen.

\section{Comparison with patients lost at follow-up}

We compared the general features of our study population ( $n=52$ ) with the group of patients lost at follow-up $(n=48)$. There was no significant difference in terms of age, tumor type and location, seizure type and frequency, and overall prognosis (with death occurring within 2 years in about $44 \%$ of cases of both groups). The chance of incomplete surgical procedure and post-surgical residue, however, was higher in the present study population compared with patients lost to follow-up (Table 2).

\section{Conclusions}

In this paper we report the long-term follow-up data on epilepsy evolution of the PERNO study in 52 patients with PBT followed up prospectively for at least two years.

Our study presents two important limitations. First of all, this study population represents only $50 \%$ of the original group [2]. However, the two groups had similar clinical and neuroradiological characteristics at study entry and identical seizure response to initial surgical treatment, therefore suggesting a comparable outcome. Indeed, the death rate after two years was $44 \%$ in both groups. Secondly, HGG were much more frequent than LGG in our population, this bias reflecting a predominance of patients from the neurooncological centers of the ER Region, as already discussed in the previous short-term study [2].

In HGG group, seizures were completely controlled in $63 \%$ of patients after a two-year follow-up, with better results (87\%) in the subgroup without tumor progression. Also in patients with neuroradiological evidence of tumor progression or evolving to death, however, complete seizure control could be achieved in about $50 \%$ of cases. Studies reporting longterm evolution of seizures in HGG are considerably rare, the main limitation being the overall dismal prognosis of these patients [3]. In partial agreement with our data, Chaichana, et al. (2009) described a seizure-freedom rate of $75 \%$ in a population of $98 \mathrm{HGG}$ cases with seizures followed up for 13.7 months [3].

In the LGG group, seizures were completely controlled in only about $10 \%$ of cases, a rate consistently lower than previously reported in other series. This finding should be considered cautiously, however, partly because the number of patients was too low (only 10 cases) and partly because these patients had gliomas with multilobar involvement, usually requiring a second surgical procedure. One possible explanation of a bad seizure prognosis in this group is that these patients underwent surgery to remove the tumor without taking into account the need to treat epilepsy. In fact, no patient underwent long-term video EEG monitoring or was discussed in a multidisciplinary team with a neurophysiologist and an epileptologist. In the literature it has been highlighted that better results in terms of seizure outcome are achieved if the surgical procedure is supplemented by pre-surgical study aimed to localize the epileptogenic area $[4,5]$. Indeed, seizure control in these patients is crucial for an improvement of quality of life [6].

As for long-term therapy in these patients, our results suggest that it is difficult to disentangle contribution of treatments from tumor evolution in the control of seizures. Surgery seems the most important factor to influence the recurrence of seizures in HGT, while the role of antiepileptic therapy is more difficult to assess. Interestingly, the most used drug was LEV in the HGT group and OXC in the LGT group, this difference probably reflecting the need to use a 
strictly non-inducing agent in HGG. Valproic acid, which has been claimed to have a robust anti-tumoral effect in addition to its antiepileptic action, is surprisingly rarely prescribed in this population [7].

We conclude that over 2-year long-term follow-up seizures seem to maintain the same semiological characteristics as observed in the short-term analysis. In particular, more than $60 \%$ of patients with HGG have complete control of seizures, the better results being observed in cases without tumor progression. In contrast, patients with HGG have refractory epilepsy, partly depending on the lack of a complete tumor removal and the lack of a presurgical neurophysiological study aimed to localize the epileptogenic area. We suggest that LGG manifesting with epilepsy should regularly undergo a detailed study of epilepsy before surgical procedure.

\section{References}

1. Kerkhof M, Vecht CJ (2013) Seizure characteristics and prognostic factors of gliomas. Epilepsia 54: 12-17.
2. Michelucci R, Pasini E, Meletti S, et al. (2013) Epilepsy in primary cerebral tumors: The characteristics of epilepsy at the onset results from the PERNO study - project of emilia romagna region on neuro-oncology. Epilepsia 54: 86-91.

3. Chaichana KL, Parker SL, Olivi A, et al. (2009) Long-term seizure outcomes in adult patients undergoing primary resection of malignant brain astrocytomas. Clinical article. J Neurosurg 111: 282-292.

4. Maschio M, Paladin F (2015) Taking care of patients with brain tumor-related epilepsy: Results from an italian survey. Neurol Sci 36: 125-130.

5. Bauer R, Ortler M, Seiz-Rosenhagen M, et al. (2014) Treatment of epileptic seizures in brain tumors: A critical review. Neurosurg Rev 37: 381-388.

6. Rahman Z, Wong CH, Dexter M, et al. (2015) Epilepsy in patients with primary brain tumors: The impact on mood, cognition, and HRQOL. Epilepsy Behav 48: 88-95.

7. Weller M, Gorlia T, Cairncross JG, et al. (2011) Prolonged survical with valproic acid use in the EORTC/NCIC temozolomide trial for glioblastoma. Neurology 77: 1156-1164.

DOI: $10.36959 / 649 / 655$ 